\title{
Augmentation of Patient Health Care Choices for Morbid Obesity by Use of Computer Decision Analysis
}

Yaw Sarpong ${ }^{1}$, Jodi Ryder ${ }^{2}$ and Scott Litofsky $\mathbf{N}^{1 *}$

${ }^{1}$ Division of Neurological Surgery, University of Missouri-Columbia School of Medicine, Columbia, Missouri, USA

${ }^{2}$ George Washington University School of Business, Washington, District of Columbia, USA

\begin{abstract}
Introduction: Many patients state that they frequently make the wrong choices when it comes to their healthcare treatment. These patients reported poor knowledge about the medical alternatives, physician biases, and lack of consideration of their goals and concerns in the treatment alternatives available led to these poor choices. However, current studies suggest that if patients are given aids that improve their knowledge and address their goals and concerns, they are able to make choices that are medically recommended as well as being right for them. We hypothesized that a computer model designed to improve knowledge and take into account patients' concerns and goals will be able to aid patients in making such decisions.

Methods: Using the Expert Choice Comparion system, we designed a program to assist morbidly obese patients in deciding which treatment options will be best suited for them. This system incorporated treatment objectives, treatment alternatives, pros and cons of each alternative, utility curves, and dynamic and performance sensitivity graphs to reach treatment recommendations. Patients were surveyed about their choices.

Results: 8 patients from a convenience sample participated in decision analysis. Most chose reduction of co-morbidities, followed by treatment safety, followed by weight loss as their primary objectives. All patients were satisfied with their choice, all 8 felt their concerns were addressed and 7 of 8 were likely to follow recommendations. The program provided them with choices that meet national guidelines. Five of 8 patients described the ease of use of the program as moderate, 2 described it as excellent, and 1 described it as poor.
\end{abstract}

Conclusions: Patients can use computer modeling to assist in making health choices for themselves.

Keywords: Morbid obesity; Patient choice; Computer decision analysis

\section{Introduction}

Theories about medical decision-making define a good medical decision as the intersection between these three points: patient values and expectation, best external medical evidence, and individual clinical expertise [1]. Often the latter two points are packaged into best-recommended healthcare practices. Normally patients have understanding about their values and expectation but lack knowledge about the latter two options. Thus, when pressed into making medical choices, patients and their family members often face difficulties in choosing between multiple options. For instance, eighty percent of American adults believed that they made the wrong decision when asked about a recent healthcare choice [2]. When queried further about these feelings, most of the responders pointed to their own limited knowledge about the medical condition, physicians' biases that guide patients' decisions, lack of physician consideration of patients' goals, and concern about treatment options as the main culprits. Consequently, a recent Cochrane Review of 86 randomized trials showed that patients' decision-making capacities are improved significantly when they are provided with aids such as educational literature, videos, and web based resources that improve their knowledge and addressed their concerns about their options. The same review found that 25 to $30 \%$ of procedures performed on patients would not be necessary if patients had the appropriate knowledge about their treatment options [2]. Further, studies by Center for Medicare and Medicaid Services (CMS) note that this behavior has led to variations in healthcare practices and contributed significantly to the increase in healthcare expenditure [3].

Computers have been used to assist physicians in clinical decisionmaking for some time in a variety of situations [4-7]. Many of these medical decision support applications have been confined to commercial interests and have not been publically available. Widespread use has not been achieved, in part because of physicians' concerns about decision accuracy, ability to integrate with workflow, lack of user friendliness, and difficulties with natural language processing [7]. Even so, formal decision analysis may support rational choice between multiple therapeutic options [6].

Similarly, a computer program designed to help patients in decisionmaking may help patients make the right decisions, receive appropriate treatment, and decrease rising cost of healthcare. One example is the Comparion system, (Expert Choice, Arlington, Virginia) which is usually used by business decision makers [8]. The Comparion system takes a problem and its possible solutions, breaks the problem into its component parts, and attempts to rectify them with the concerns and goals of the user. It then provides a solution that will meet the users' goals and concerns [8]. We hypothesized that use of the Comparion system would enhance patients' abilities to make choices for their own health care. In order to test this hypothesis, we selected a group of patients with frequent frustration with their health care choices - those with

*Corresponding author: Scott Litofsky N, Division of Neurological Surgery, University of Missouri-Columbia School of Medicine, One Hospital Drive, MC 321 Columbia, Missouri 65212, USA, Tel: 573-882-4909; Fax: 573-884-5184; E-mail: litofskyn@health.missouri.edu

Received May 19, 2014; Accepted June 10, 2014; Published June 14, 2014

Citation: Sarpong Y, Ryder J, Litofsky NS (2014) Augmentation of Patient Health Care Choices for Morbid Obesity by Use of Computer Decision Analysis. J Comput Sci Syst Biol 7: 144-148. doi:10.4172/jcsb.1000149

Copyright: (c) 2014 Sarpong Y, et al. This is an open-access article distributed under the terms of the Creative Commons Attribution License, which permits unrestricted use, distribution, and reproduction in any medium, provided the original author and source are credited. 
morbid obesity. Obesity, defined as Body Mass Index (BMI= weight in $\mathrm{kg} /$ height in $\mathrm{cm}^{2}$ ) greater than 30 [9] is estimated to be present in $35.7 \%$ of adults and $16.9 \%$ of children and adolescents in the United States [7]. In morbid obesity, BMI is greater than 40 . Because obese patients frequently have other co-morbidities, including cardiovascular disease, hypertension, Type II diabetes, sleep apnea, cancers, hyperlipidemia, esophageal reflux, anxiety, depression, posttraumatic stress disorder (PTSD), binge eating, and night eating syndrome [10], they have high medical system utilization and contribute to the healthcare deficit. An obese patient's healthcare costs is approximately 42 percent greater than a normal weight individual and total medical care of obese patients costs 147 billion dollars per year [11]. Obese people, on average, live 10 years shorter than normal weight individuals [11]. In addition to health risks, obesity is also associated with social dysfunction, with obese individuals more likely to be single, disrespected, passed over for a promotion, and has lower paying jobs. Additionally, society treats obese individuals as weak willed and lazy, and attempts to isolate and marginalize them [11].

To address the medical and social ills associated with obesity, numerous treatments are available. Non-surgical treatments include dieting and exercising. Surgical treatments consist of Roux-en Y gastric bypass (RYGB), Sleeve Gastrectomy (SG), and Adjustable Gastric Banding (ABG) [10]. Each treatment has its own weight loss efficacy, complications, and resolution of co-morbidities. These complexities can contribute to obese patients feeling confused, overwhelmed, and discouraged as they attempt to make choices for their health care; in hindsight, many suggest that they picked the wrong treatment options. The objective of this study is to show that the Comparion program can help each morbidly obese individual navigate through a set of treatment alternatives to make a health care choice that will help him/her meet his/her personal treatment goals.

\section{Materials and Methods}

To design the program, a domain was obtained on the Expert Choice Comparion website, which we entitled "Morbid Obesity Choice Model." The program is designed to allow the programmer to input information regarding objectives, alternatives, and pros and cons of each alternative option. Using this design in the Comparion website, the domain was programmed with treatment objectives for morbid obesity, alternative treatment choices, and pros and cons of each treatment choice. Utility curves denoting the rates at which each treatment option meets the objective of treating obesity and its co-morbidities were assigned to each treatment alternative. These fields were developed as described below. Once the program was completed, patients were asked to log in via user name and password created for them to answer questions regarding goals and concerns in treatment for their morbid obesity. The program then rectifies the answers with its base knowledge concerning the treatment alternatives, efficacy, risks, and benefits. The program uses this information to develop Dynamic and Performance Sensitivity graphs, which compared each the treatment alternative to the others in terms of how they meet each patient's goals. The patients could then choose the treatment option that best suited their goal of treatment.

\section{Objectives}

Based on recommended bariatric treatment guidelines from the National Institutes of Health (http://www.nhlbi.nih.gov/guidelines/ obesity/prctgd_c.pdf), goals of weight reduction surgery are defined as follows: 1) weight loss, 2) reduction of co-morbidities, and 3) safety of treatment without significant risk [12-14]. Furthermore, the guidelines divided the objective of reduction of co-morbid conditions into subobjectives that included: 1) cure reflux, 2) treatment of Type 2 diabetes, 3) reduction of hyperlipidemia, 4) resolution of hypertension, and 5) treatment of sleep apnea. These objectives were programmed into the Comparion domain with 3 main objectives and the sub-objectives under the reduction in co-morbidities objectives and are outlined in Table 1.

\section{Alternatives}

The guidelines for bariatric treatment divide treatment alternatives for morbid obesity into non-surgical and surgical treatment options. The non-surgical alternatives consisted of diet and exercise. On the other hand, the surgical alternatives consisted of Adjustable Gastric Banding (ABG), Sleeve Gastrectomy (SG), and Roux-en-Y Gastric Bypass (RYGB) [12-14]. The alternatives with its sub-alternative components were programmed into Comparion domain as well (Table 1).

\section{Pros and cons}

In the bariatric treatment guidelines, there are pros and cons attributed to each treatment alternatives to provide. These pros and cons were built into the Comparion domain. Pros and cons for diet and exercise, RYGB, SG, and ABG are listed in Table 1.

\section{Utility curve}

The bariatric treatment guidelines also provided percentage rates at which each treatment options have historically achieve the treatment objectives [12-14]. These rates were input into the program via a

\begin{tabular}{|c|c|c|c|}
\hline Alternatives & Sub Alternatives & Pros & Cons \\
\hline Non-surgical & Diet and exercise & No surgical risks & $\begin{array}{c}\text { High failure rate } \\
\text { Does not cure reflux } \\
\text { Does not treat sleep apnea } \\
\text { Ineffective at Type } 2 \text { Diabetes treatment }\end{array}$ \\
\hline \multirow[t]{3}{*}{ Surgical } & Adjustable gastric banding (ABG) & $\begin{array}{c}\text { Weight loss } 40.5 \% \\
\text { Type } 2 \text { Diabetes cure } 47.8 \% \\
\text { Hyperlipidemia cure } 78 \% \\
\text { Sleep apnea cure } 94.5 \%\end{array}$ & $\begin{array}{c}\text { Worsens reflux } \\
\text { Surgical Risks }\end{array}$ \\
\hline & Sleeve gastrectomy (SG) & $\begin{array}{c}\text { Weight loss } 66 \% \\
\text { Type } 2 \text { Diabetes cure } 56 \% \\
\text { Hyperlipidemia cure } 77 \% \\
\text { Sleep apnea cure } 60 \%\end{array}$ & $\begin{array}{c}\text { Surgical risks } \\
\text { Does not cure reflux } \\
\text { Does not cure hypertension }\end{array}$ \\
\hline & Roux-en-Y gastric banding (RYGB) & $\begin{array}{c}\text { Weight loss } 62 \% \\
\text { Type } 2 \text { Diabetes cure } 78 \% \\
\text { Hyperlipidemia cure } 61 \% \\
\text { Reflux cure } 100 \% \\
\text { Hypertension cure } 66 \%\end{array}$ & Surgical risks (generally low) \\
\hline
\end{tabular}

Table 1: Programmed alternatives with its sub-alternative components into Comparion domain. 
utility curve that accurately assigned the historical percentages to each treatment alternatives in regards to objectives.

\section{Study population}

Patients were randomly selected from a group of patients who were contemplating treatment for morbid obesity at Chesapeake Regional Medical Center in Virginia. These patients were approached about the technology and eight patients randomly agreed to participate and give consent for the project. All of these patients had a BMI of 35 or greater with or without co-morbidities. Further, they had participated in a Medical Center-sponsored didactic course designed to educate patients about the NIH guidelines for morbid obesity treatment. These patients were given tutorial on the Comparion program and how to $\log$ on and interact with it. The patients were then given username and password for the Comparion domain after they were given access to domain by the researchers. Once they logged on, the program guided the patients through question fields regarding their goals and concerns in contemplating treatment for morbid obesity. The program tabulated the results from their evaluation (Figure 1) and created a graph that provided patients with alternatives that could meet their goals and address their concerns. The choices offered to patients were subsequently compared to the nationally recommended treatment guidelines for patients in this $\mathrm{BMI} / \mathrm{co}$-morbidities group, which was malabsorptive surgery using RYGB [12]. Patients were then surveyed to rate their satisfaction regarding the choices provided to them by the program, if their choices met their goals and addressed their concerns, the ease of usability of the program, and how likely are they to follow through with the recommendations.

\section{Results}

\section{Treatment choices}

After the patients interacted with Morbidly Obese Choice Model, the program calculated the results and derived arithmetic percentages for success regarding treatment alternatives to objectives for each patient. Choices differed between patients. For instance, patient 1 , 3 and 7 rated reducing co-morbidities as most important objective followed by weight loss and safety/surgical risks. In contrast, Patients 2 and 8 rated weight loss as most important followed by reduction in comorbidities and safety/surgical risks. Patients 4, 5 and 8 rated reduction in co-morbidities as number one followed by safety/surgical risks and weight loss. Finally, patient 6 rated safety/surgical risks as most important followed by weight loss and reduction of co-morbidities. These results are summarized in Figure 1.

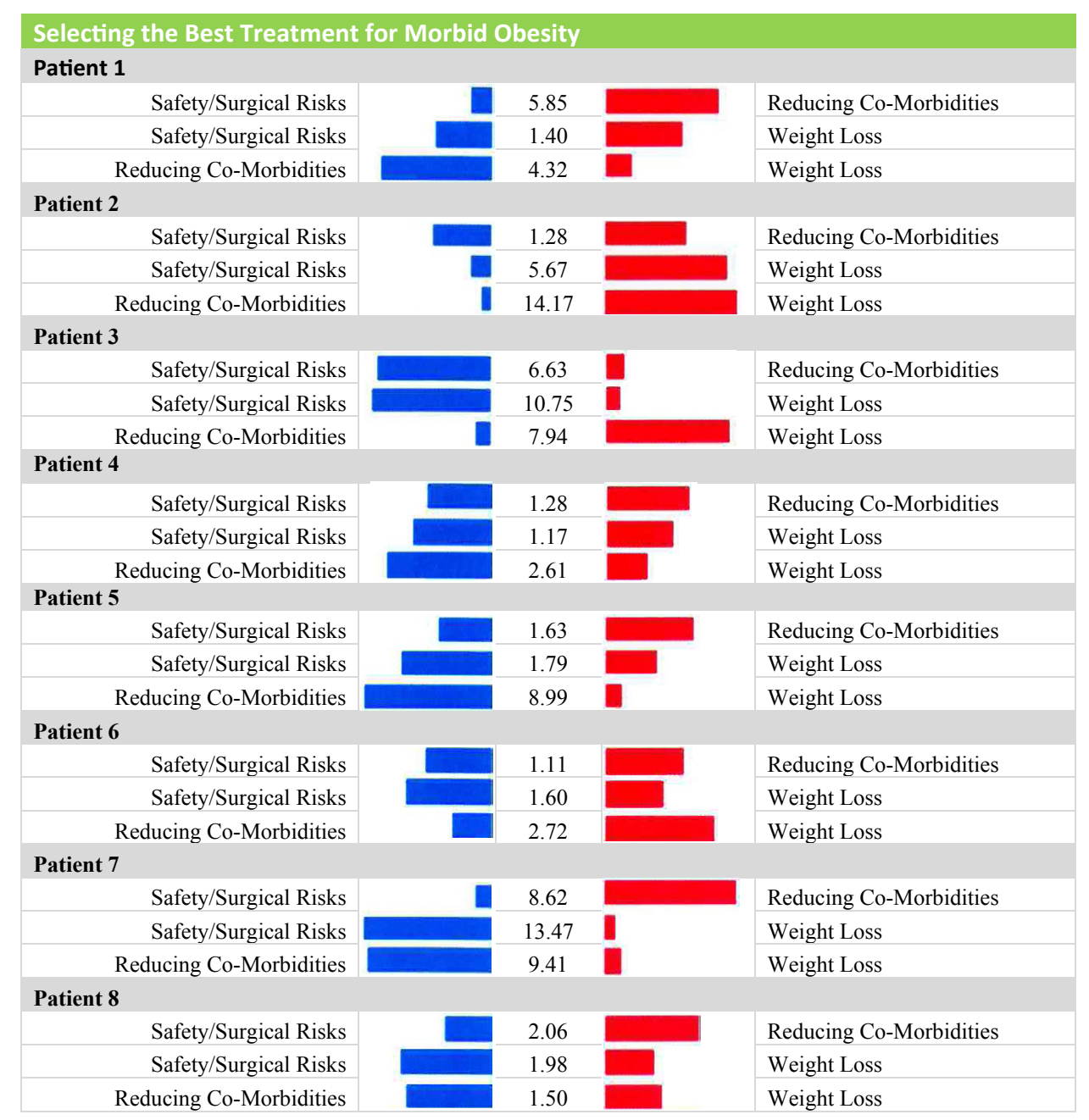

Figure 1: Study population choices of preferred objectives of treatment of obesity.

Each patient's preference for objective of treatment are listed by weighted importance to the patient. This graph is derived from Program print-out. 
In aggregate, the patients rated reduction in co-morbidities as their number one objective followed by weight loss with safety/surgical risks as a distant third. Further, they rated RYGB as the best alternative followed by SG, then AGB, and diet and exercise as the last of the alternatives (Figure 2). Based on analysis of these objectives and the ability of each alternative to achieve the objectives, the Comparion program then produced a performance sensitivity graph (Figure 3) which compared how well the treatment alternatives met the stated objectives of treatment. In essence, this graph is the summary of the aggregate choice of treatment for the participating patient population. The patient population in this study felt diet and exercise was the best option in meeting the objective safety/surgical risks while RYGB was felt to meet the objective of reduction in co-morbidities. SG was the modality thought to meet the weight loss objective. Through the computer analysis, RYGB was rated as best option in meeting all the objectives, followed by SG, then AGB, and lastly, diet and exercise. The rating of the RYGB as the best treatment option for this group compares favorably with nationally recommended treatment guidelines for this patient group [12].

\section{Patient surveys}

All 8 patients felt that choices provided for them by the program were satisfactory. All 8 patients also felt that the choice provided by the program addressed their concerns and met their overall goals. Seven out of 8 patients stated that they would definitely use the

\section{Objectives}

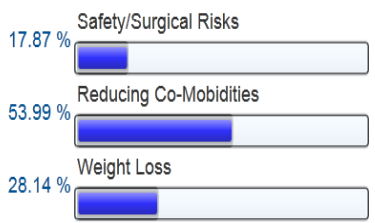

Figure 2: Dynamic Sensitivity Graph.

The aggregated preferences of the objectives of treatment for the study population as a whole are expressed as percentages. The percentages for the alternatives represent the collective proportion of choice for the entire group to obtain their preferred objects. This graph is derived from Program print-out.

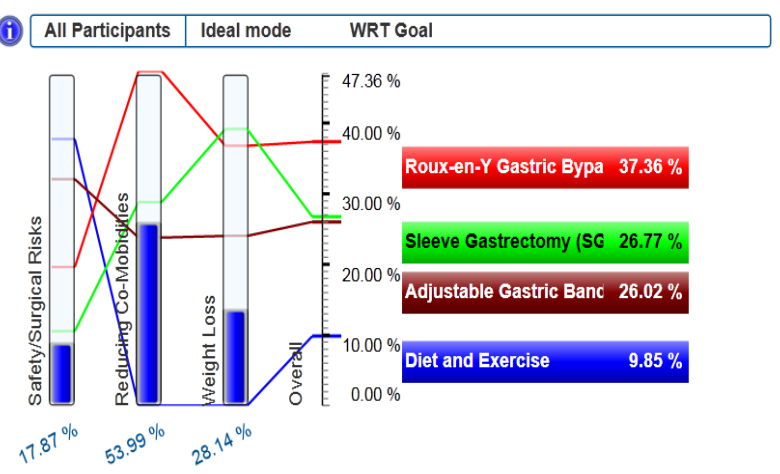

Figure 3: Performance Sensitivity Graph

This graph shows the ability of the different alternatives to meet each objective based on the utility curves which accounted for the historically success rate of each treatment option. This graph is derived from Program print-out. recommendation provided by the program as a treatment option for their morbid obesity (Figure 4). The one patient who stated that he would not use the computer choices decided that he preferred to get his recommendations from another human being instead of a machine. In the patient survey regarding usability of the program, two patients rated the program as excellent, five rated it as moderate, and one rated as poor - this patient subsequently decided to not use the program's recommendation (Figure 5).

\section{Discussion}

Based on the current theories in best medical management [1], best treatment options are those that combine the patient's values and concerns with nationally recommended guidelines for treatment. This study showed that patients could use computer-assisted decisionmaking tools to make these treatment decisions. In the study, the program providing assistance to patients consists of the treatment objectives and alternatives, as well as the pros and cons of the treatment alternatives, thereby aiding patients in making informed decisions.

These informed decisions are unlike the informed decisions patients are faced with in daily medical practices as they are not biased by physicians' perception and interpretation of data. In addition, this patient-based computer-assisted decision making can provide physicians with additional insights as they care for their patients,

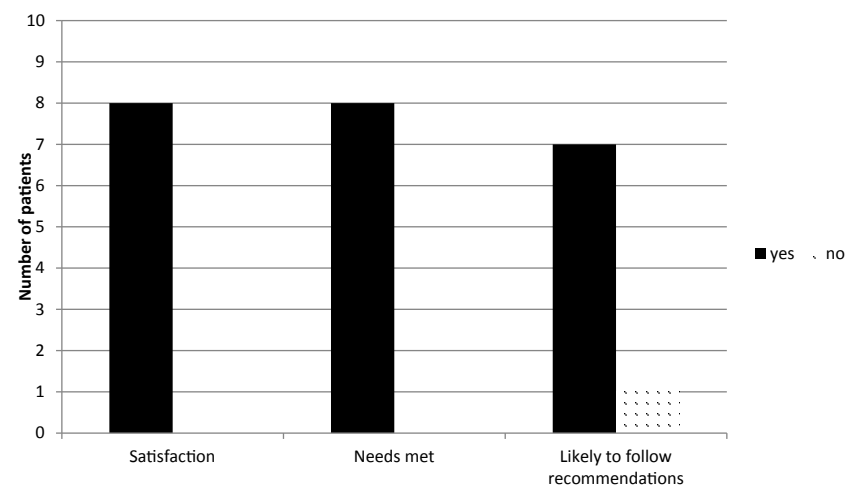

Figure 4: Patient Survey Results Regarding the Use of the Morbidly Obese Choice Model

This graph arranges the result of the patient survey into a visual format. The graph denotes patient satisfaction with the program, whether the program met patient needs, and whether the patient is likely to follow the recommendations by either yes or no responses.

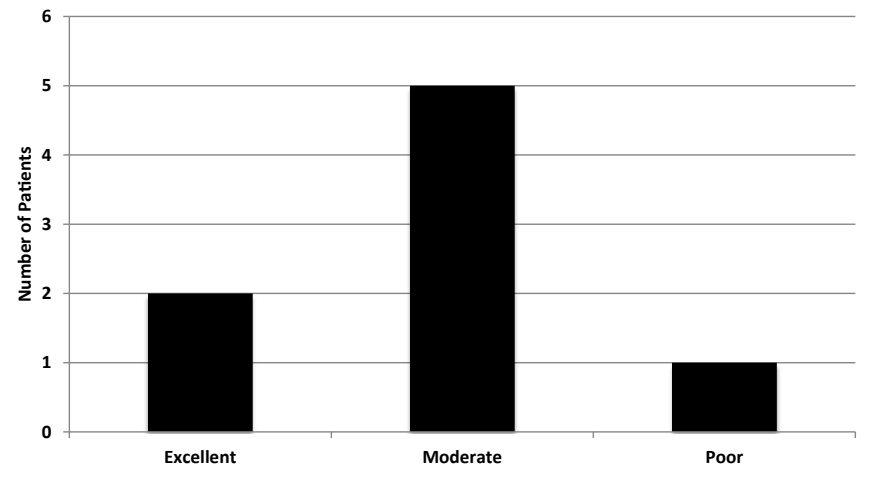

Figure 5: Graph comparing how the patients rated the usability of the program This graph illustrates the patients' self-ratings of the ease of usability of the Comparion program as either excellent, moderate, or poor after using the program. 
because the physicians will know that the plan was based on the patient's goals and objectives as well as the nationally recommended guidelines. Furthermore, this program could serve as an avenue for patients to gain knowledge about their disease, treatment alternatives, and possible goals of care. As such, computer-assisted patient decision-making can improve communication avenues between physicians and their patients in the course of treatment of an illness.

The findings in this study can help shift the current paradigm regarding the use of computer-assisted support in medical decisionmaking in a number of ways. In this study, computer support from the use of probability and equations to use of current medical knowledge to help develop concrete solutions for patients and physicians. Further, it shifts use of computer modeling decision systems to prevent physician error to use of these systems to help physicians provide improve quality of care for their patients and give patients autonomy. In contrast to previous computer support, this system shifts the computer modeling in medical decision-making paradigm because it builds a system that was designed primarily for patients rather than physicians. Lastly, it provides an avenue by which patients can be educated about medical issues, treatment alternatives, and risks and benefits inherent in treatment options without biases or differing interpretations of their providers.

Although this study does provide support for the concept of computer-assisted medical decision-making for patients and physicians, the study has several limitations. The small sample size is limiting because of lack of statistical power to conclude that the study results support the hypothesis. Additionally, this study was performed on the beta design of the program and is not based on the finished program; interpretation and outcomes may be somewhat erratic given that the program needs further fine-tuning. The results seen here with computer-assisted decision-making may not be applicable to other disease states. Patients who prefer a more paternalistic physician relationship may not react well to the programing methodology. Many patients may not be computer savvy and may struggle with information input and retrieval. Incorporation of this type of system into the physician-patient interaction may be time consuming and reduce efficiency in the work-flow of the hospital or clinic.

These limitations notwithstanding, the results show that computerassisted patient decision-making is possible and potentially beneficial. Further efforts in computer-assisted patient choice are warranted. Future directions include program development to help patients improve knowledge and understanding of best treatment options for a variety of different diseases. Larger patient samples are necessary to validate such work. Modifications in the program are necessary to improved ease of use and allow patients to access it from the comfort of their own phone or home.

\section{Conclusions}

The Morbidly Obese Choice Model using the Expert Choice Comparion program illustrates how computer-assisted decisionmaking programs can take different objectives, alternatives, risks and benefits inherent in medical treatment options and rectify them with patients concerns and goals to assist patients in making health care choices which better satisfy their needs.

\section{Acknowledgements}

The authors wish to thank Barb Klund of the Division of Neurological Surgery at University of Missouri-Columbia School of Medicine for her assistance with preparation of the manuscript.

\section{References}

1. Gray JAM (1997) Evidence-based healthcare: how to make health policy and management decisions. London: Churchill Livingstone.

2. Stacey D, Légaré F, Col NF, Bennett CL, Barry MJ, et al. (2014) Decision aids for people facing health treatment or screening decisions. Cochrane Database Syst Rev 1: CD001431.

3. Hostetter M, Klein S (2012) Quality Matters Helping Patients Make Better Treatment Choices with Decision Aids.

4. Hansen MV, Zdanowski A (1997) The urological management of men with lower urinary tract symptoms, recorded using an interactive computer program. Br J Urol 80: 205-210.

5. Klar R, Bayer U (1990) Computer-assisted teaching and learning in medicine. Int J Biomed Comput 26: 7-27.

6. Van Dessel E, Fierens K, Pattyn P, Van Nieuwenhove Y, Berrevoet F, et al (2009) Defining the optimal therapy sequence in synchronous resectable liver metastases from colorectal cancer: a decision analysis approach. Acta Chir Belg 109: 317-320

7. Wagholikar KB, Sundararajan V, Deshpande AW (2012) Modeling paradigms for medical diagnostic decision support: a survey and future directions. J Med Syst 36: 3029-3049.

8. http://expertchoice.com

9. Ogden CL, Carroll MD, Kit BK, Flegal KM (2012) Prevalence of obesity in the United States, 2009-2010. NCHS Data Brief 1-8.

10. Finkelstein EA, Trogdon JG, Cohen JW, Dietz W (2009) Annual medical spending attributable to obesity: payer-and service-specific estimates. Health Aff (Millwood) 28: w822-831.

11. Diamond $L$ (2010) Links between obesity and mental health.

12. NHLBI Obesity Education Initiative. The practical guide identification, evaluation, and treatment of overweight and obesity in adults.

13. Morning M (2012) For people who are extremely overweight and likely to develop diabetes, surgery may be the best form of prevention. Monday Morning 20: 1.

14. Smith BR, Schauer P, Nguyen NT (2011) Surgical approaches to the treatment of obesity: bariatric surgery. Med Clin North Am 95: 1009-1030. 